\title{
Th2 cells and GATA-3 in asthma: new insights into the regulation of airway inflammation
}

Perspective

See related articles, pages: 995-1006.

\author{
Anuradha Ray and Lauren Cohn \\ Section of Pulmonary and Critical Care Medicine, Yale University School of Medicine, New Haven, Connecticut 06520, USA \\ Address correspondence to: Anuradha Ray, Pulmonary and Critical Care Medicine, Department of Internal Medicine, \\ Yale University School of Medicine, 333 Cedar Street, 105 LCI, New Haven, Connecticut 06520-8057, USA. Phone: (203) 737-2705; \\ Fax: (203) 785-3826; E-mail: Anuradha.Ray@yale.edu.
}

Asthma is a clinical syndrome characterized by intermittent episodes of wheezing and coughing. The diagnosis is confirmed by abnormal lung physiology, including reversible airway obstruction and airways hyperresponsiveness (AHR). The pathologic manifestations of asthma include airway inflammation, airway remodeling, and mucus hypersecretion. Since the advent of bronchoscopy, we have been able to assess the lower airways in asthmatics during periods of disease activity and when the disease is quiescent and lung function is normal. From these studies, it has become clear that persistent inflammation is central to the pathogenesis of asthma. Bronchoscopic biopsies, bronchoalveolar lavage (BAL), and bronchoprovocation studies in patients with allergic asthma, intrinsic (nonallergic) asthma, or isocyanate-induced asthma show similar characteristic inflammatory responses. Increased numbers of inflammatory cells, predominantly eosinophils and lymphocytes, infiltrate the mucosa and submucosa in asthmatics independent of the etiology. It is now believed that airway inflammation leads to AHR (reviewed in ref. 1), airway remodeling (see article by Elias et al. in this series), and mucus hyperplasia $(2,3)$.

Our objective in this review is to define the role of $\mathrm{CD}^{+}$ Th2 cells in asthma and to detail the cellular and molecular mechanisms involved in the generation of Th2 cells in the respiratory tract. By outlining the steps that occur in Th2 cell differentiation, recruitment, and activation in the lung, we will define novel points at which we may be able to direct immunologic and pharmacologic interventions in the treatment of this disorder.

$\mathrm{CD}^{+} \mathrm{T}$ cells in asthma. $\mathrm{CD} 4^{+} \mathrm{T}$ cells are believed to play a crucial role in controlling inflammation in asthma. They make up the predominant lymphocyte population that infiltrates the airways in asthmatics, and are activated in these sites, expressing the surface activation markers MHC class II and CD25 (IL-2R) (4, 5). Although CD4 ${ }^{+} \mathrm{T}$ cells make up only a fraction of leukocytes in the lung, they are critically important for adaptive immunity in normal individuals. They have the ability to rapidly expand in response to specific stimuli, and through the release of cytokines, $\mathrm{CD}^{+} \mathrm{T}$ cells communicate with other cells, initiating cascades of inflammatory mediators for effective host defense. In addition to $\mathrm{CD} 4^{+} \mathrm{T}$ cells, $\mathrm{CD}^{+} \mathrm{T}$ cells and $\gamma / \delta \mathrm{T}$ cells have been identified in the airways of asthmatics (5), but their roles in orchestrating pulmonary immune responses are less clear. $\mathrm{CD}^{+} \mathrm{T}$ cells can also be memory cells, retaining the ability to respond to an antigen for the lifetime of the host. Thus, for all of these reasons, $\mathrm{CD} 4^{+} \mathrm{T}$ cells are believed to initiate and perpetuate the inflamma- tory response that is characteristic in asthma.

Association of $\mathrm{CD}^{+}{ }^{+} \mathrm{Th} 2$ cells and asthma. Antigen-activated $\mathrm{CD}^{+} \mathrm{T}$ cells can differentiate into effector cells that possess distinct functional properties conferred by the cytokines they secrete $(6,7)$. Th 1 cells are a subset of $\mathrm{CD}^{+}$ T cells that secrete the macrophage-activating factor IFN$\gamma$ and lymphotoxin, whereas $\mathrm{CD}^{+} \mathrm{Th} 2$ cells produce a different panel of cytokines, including IL-4, IL-5, and IL-13 (8, 9). Th1 cells stimulate strong cell-mediated immune responses, particularly against intracellular pathogens. Th2 cells, through the production of IL- 4 and IL-13, are potent activators of B-cell antibody production, particularly IgE (10). IL-5 secretion by Th2 cells is critical for eosinophil differentiation and maturation $(11,12)$.

In asthmatics, $\mathrm{CD}^{+} \mathrm{T}$ cells producing IL-4, IL-5, and IL13 have been identified in BAL and airway biopsies. These cytokines are secreted in the airways of patients with mild or asymptomatic disease $(5,13)$. After antigen challenge in allergic asthmatics, Th2 lymphocytes are increased in the airways of patients $(1,5,13)$. Because Th 2 cells have been identified in the airways of asthmatics, and because Th2 cytokines are required for the development of airway eosinophilia and IgE, it has been proposed that Th2 cells stimulate an inflammatory response that results in asthma.

The hypothesis that Th2 cell activation leads to the development of asthma has caused debate, as some studies failed to show typical Th2 cytokine patterns in the airways of asthmatics. In nonallergic (intrinsic) asthmatics, for example, IL-5, but not IL-4, protein was detected in BAL fluid and in supernatants from BAL lymphocytes (5). Yet, in allergic asthmatics, both IL-4 and IL-5 were produced. This variable expression of Th2 cytokines suggested that different cytokines could stimulate the pathologic changes in asthma. More recent studies show that mRNA for IL-13 is expressed in $\mathrm{CD}^{+}$ cells in airway biopsies of both allergic and nonallergic asthmatics $(14,15)$, suggesting that a "true" Th2 phenotype characterizes asthma independent of the etiology.

Th1 cells were also identified in the airways of asthmatics, and were speculated to play a role in the pathogenesis of the disease (16). When activated nonspecifically, BAL cells recovered from allergic asthmatics produced IFN- $\gamma(17,18)$. Yet, bronchoprovocation with allergen did not lead to an increase in IFN- $\gamma$ production (19), indicating that the Th1 cells present in the airways of asthmatics are not allergen-specific. They may be bystanders in the airways, responding to other antigens or, alternatively, functioning in a regulatory capacity.

Th2 cells induce characteristic features of asthma in animal 
studies. Whereas investigations in humans highlight an association of Th 2 cells and asthma, animal studies show cause and effect. Many studies have shown that eosinophilic airway inflammation and AHR are dependent upon $\mathrm{CD}^{+} \mathrm{T}$ cells $(1,12)$. In addition, adoptive transfer of $\mathrm{CD}^{+} \mathrm{T}$ cells from animals with antigeninduced AHR resulted in airway inflammation and hyperresponsiveness in aerosol-challenged recipient mice (20). The ability of Th2 cells to stimulate the characteristic features of asthma was recently shown. When antigen-specific Th1 or Th2 cells were generated in vitro, transferred into recipient mice, and activated in the respiratory tract with inhaled antigen, Th2 cells induced airway eosinophilia, mucus hypersecretion, and AHR (2, 21). Th1 cells resulted in a neutrophil-predominant inflammatory response without any of the characteristic features of asthma. Thus, Th2 cells can activate inflammatory pathways that result in airway inflammation and AHR after short-term exposure to antigen. Constitutive production of individual Th2 cytokines has also been found to induce an asthma-like syndrome. Transgenic mice that overexpress the Th2 cytokines - IL-4, IL5, IL-13, and IL-9 - in the airway epithelium exhibit common inflammatory features in the airways, including eosinophilia and mucus overproduction. Transgenic mice that overexpress IL-13, IL-9, and IL-5 showed AHR and collagen deposition in the airways, indicating that chronic exposure to Th2 cytokines can also induce airway remodeling (see article by Elias et al. in this series). Therefore, it appears that activation of Th2 cells is sufficient for the induction of inflammation and the chronic pathologic changes associated with asthma.

Animal studies have now demonstrated how individual Th2 cytokines control different features of the inflammatory response in asthma. IL-5 production is essential for airway eosinophilia (12), and, in some cases, AHR depends on eosinophilia (1). Recent studies have shown that the induction of AHR, eosinophilia, and mucus production by Th2 cells requires signaling through IL-4R (1). This appears predominantly to reflect the role of IL-13 in these processes $(22,23)$, because in the absence of IL-4, AHR, lung eosinophilia, and mucus production can still be induced $(2,21)$. Whether IL-4 can also stimulate these asthmatic phenomena in the absence of IL-13 has not yet been shown. IL-4 is critical for Th2 cell induction, and IL-4 may be required for the persistence of Th2 cells in vivo in the lung (24).

Th2 cytokines can induce a constellation of pathophysiologic changes in animals that resemble human asthma. But, the production of IL-4, IL-5, and IL-13 is not exclusive to $\mathrm{CD}^{+} \mathrm{T}$ cells. In fact, $\mathrm{CD}^{+}$and $\gamma / \delta \mathrm{T}$ cells, and eosinophils, mast cells, and basophils have all been shown to produce these cytokines. Of all of these inflammatory cells, CD4 ${ }^{+} \mathrm{T}$ cells are typically present in higher numbers in the respiratory tract, thus explaining why the pathophysiologic abnormalities depend on $\mathrm{CD}^{+} \mathrm{T}$ cells in many animal models of asthma. But, in certain animal systems, eosinophils, mast cells, and CD8 or $\gamma / \delta \mathrm{T}$ cells have been shown to contribute to airway inflammation and $\operatorname{AHR}(25,26)$. Different animal strains or variations in methods for induction of airway inflammation may accentuate the effects of one cell type or another. Thus, these divergent results may indicate that the consequences of inflammation in a particular animal model depend on a non-CD4 ${ }^{+} \mathrm{T}$ cell that is contributing Th2 cytokines. What appears to be clear at present is that the secretion of Th2 cytokines is critical for the induction of the characteristic airway inflammation in animal models of asthma. In humans, Th2 cells are present and actively secreting cytokines in the airways. These cytokines influence the inflammatory response and likely lead to the pathophysiologic changes associated with asthma.

Th2 cell generation. Naive $\mathrm{CD}^{+}{ }^{+} \mathrm{T}$ cells encounter antigen in the lymph node, where they are stimulated to differentiate along 1 of 2 pathways: Th1 or Th2 (Figure 1). Once matured, these effector Th cells can be called into an area of inflammation, such as the lung, where they produce cytokines, resulting in the initiation of cascades of inflammatory mediators. After performing their secretory function, effector Th1 or Th 2 cells are believed to have 1 of 2 fates. They may either undergo cell death or become part of a memory cell population. $\mathrm{CD}^{+}$memory $\mathrm{T}$ cells are long-lived, maintain their polarity as Th1 or Th2 cells, and thus can be restimulated with specific antigen to secrete the same cytokines as they did previously. Once an antigen-specific, Th2-dominated response is established in the lung, it may persist for the life of the host.

Cellular basis of Th2 cell induction in the respiratory tract. A variety of different factors have been shown to control

\footnotetext{
Figure 1

Generation of Th 1 and Th2 cells from a naive $C D 4^{+} T$ cell. A naive CD4 ${ }^{+}$ $T$ cell does not secrete cytokines, and has low levels of GATA-3 and cMaf expression. Differentiation along the Th1 or Th2 pathway is triggered by stimulation with antigen $(\mathrm{Ag})$, presented to the $\mathrm{T}$-cell receptor in the context of $\mathrm{MHC}$ by the appropriate APC, and by a second signal, imparted by ligation of costimulatory molecules B7-1/B7-2 and CD28. In the lung, DCs represent the key APC. The DCs have been classified into 2 subsets: mature (DC1) and immature (DC2). The mature DCs express high levels of MHC class II on their surface and produce IL-12, which drives Th1 differentiation. The immature DCs express low levels of MHC class II and produce IL-10, which favors Th2 differentiation. Furthermore, the cytokine(s) present in the microenvironment, IL-4/IL10 versus IL-12, plays a decisive role in orchestrating the differentiation along the Th1 or Th2 lineage.
}

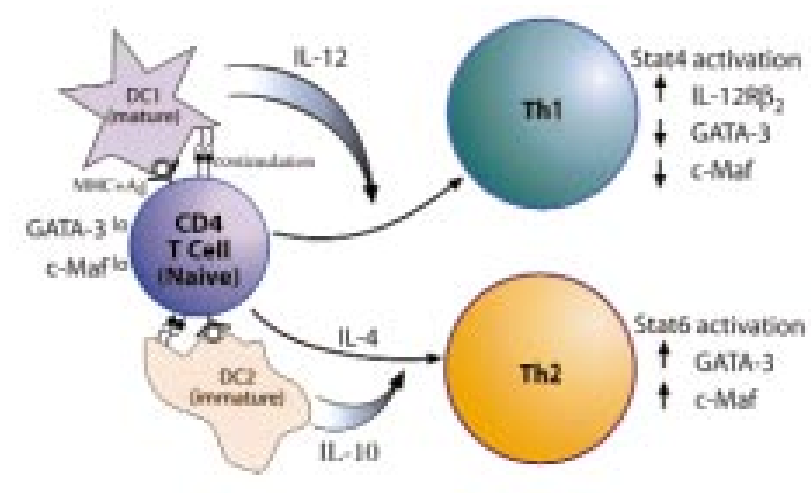


whether naive $\mathrm{CD} 4^{+} \mathrm{T}$ cells develop into Th1 or Th2 cells. The most potent signals in influencing this process are cytokines. If $\mathrm{CD}^{+} \mathrm{T}$ cells are activated by antigen/antigenpresenting cells (APCs) in the presence of IL-12, a Th1dominated population will develop, whereas if IL-4 is present in the environment, Th2 cells are induced. In the respiratory tract, many studies now suggest that Th2 responses are preferentially stimulated. The cells that line the bronchial airways create the cytokine microenvironment that controls $\mathrm{CD}^{+}$Th subset differentiation. Mast cells secrete IL-4, IL-5, and IL-13 in response to cross-linking of IgE by antigen or by non-IgE-mediated mechanisms. In this manner, the early production of cytokines may initiate a shift in the immune responses toward Th2. Lymphocytes residing near the respiratory tract and secreting IL-4 - including NK1.1, $\gamma / \delta$ T cells, and activated Th2 cells - may also influence the cytokine milieu and shift a T-cell response toward Th2.

Dendritic cells (DCs), the predominant APC in the lung, have also been shown to promote Th2 cell development in the respiratory tract. DCs form a network within the airway mucosa where they take up and process inhaled antigens and then migrate to local lymph nodes to present the processed antigen to $\mathrm{CD}^{+} \mathrm{T}$ cells. Recent studies indicate that respiratory tract DCs express low levels of surface MHC class II, and produce IL-10 but minimal IL-12 - an immature phenotype that favors Th2 cell differentiation (27). These mucosal surface DCs, alternatively termed DC2, preferentially stimulate Th2 cell development in an IL-4-independent fashion (28). In contrast, mature DC1 cells isolated from peripheral lymphoid organs stimulate the generation of Th1 cells. Th1/Th2 differentiation may also be influenced by the interactions of costimulatory molecules on APCs with their ligands on $\mathrm{CD}^{+} \mathrm{T}$ cells. Studies in other organs have shown the importance of CD40-CD40L and CD80/CD86-CTLA-4/CD28 interactions in Th2 generation (29). Elevated expression of both CD80 and CD86 has been shown on APCs in asthmatics. In the respiratory tract, these cell-surface interactions have not yet been shown to control the polarization of $\mathrm{CD}^{+} \mathrm{T}$ cells toward Th1 or Th2, but these molecular interactions are important for activation of naive $\mathrm{CD} 4^{+} \mathrm{T}$ cells and for recall $\mathrm{Th}$ responses. Lastly, some aeroallergens, including the dust mite antigen Der $\mathrm{p} 1$, have been observed to possess proteolytic activity that may shift responses in the lung toward Th2, either through effects on the cytokine milieu or on IgE regulation (30).

Th1 responses can be generated in the respiratory tract when the appropriate stimulus is provided. For example, pathogenic organisms that require macrophage activation for host defense, such as Mycobacterium tuberculosis, stimulate IL-12 production by APCs, resulting in a Th1dominated, cell-mediated immune response.Generally, the respiratory tract appears to have immunologic controls that favor Th2 responses and thus may protect the lung and preserve gas exchange by inducing humoral responses rather than highly cellular responses that lead to cell-mediated injury. In addition, Th2 cells activate other noncellular mechanisms of host defense, such as mucus production. Asthmatic individuals exhibit the negative effects of these lung-protective immune responses: chronic Th2 cell-induced inflammation in the respiratory tract, mucus production, and recurrent episodes of airway obstruction.

Molecular basis of the Th2 response. Although many different factors have been shown to control CD4 $4^{+}$Th subset differentiation, the mechanism(s) by which these factors regulate Th1/Th2 cell generation is poorly understood. Recent studies, however, have demonstrated that differential cytokine production by Th1 and Th2 cells is regulated at the level of cytokine gene transcription. They also demonstrate that this regulation involves an interplay between chromatin structure, tissue-specific transcription factors, and probabilistic gene activation. Each is reviewed below.

Role of chromatin remodeling. A sequential 2-step model involving differential programming in Th1 and Th2 cells has been suggested by Agarwal and Rao to explain specific cytokine gene expression in T-cell subsets (31). In this model, naive $T$ cells have a closed chromatin configuration around the IL-4/IL-5/IL-13 and IFN- $\gamma$ gene loci (Figure 2). In the first step, after cells are induced to differentiate by antigen and cytokine, rapid chromatin remodeling and CPG demethylation occur, with the acquisition of a characteristic cell-specific open chromatin structure. This leads to occupancy of the accessible DNA by Th2-specific transcription factors such as GATA-3 and c-Maf in Th2 cells. In the second step, these tissue-specific transcription factors synergize with transiently induced factors that are more widely expressed, such as AP-1 and NF-AT, to induce transcription of Th2 genes. It appears that the open chromatin configuration and the expression of tissue-specific transcription factors are maintained in the polarized cells. During subsequent antigenic stimulation of the memory/effector cells, transcription factors such as AP-1 are rapidly induced and synergize with preexisting factors such as GATA-3 and c-Maf, causing active transcription of the IL4/IL-5/IL-13 gene locus in Th2 cells. A similar model can be envisaged for Th1 gene expression, although much less information is presently available for Th1-specific gene expression. Some of the key transcription factors in Th2specific gene expression are discussed below.

\section{Transcription factors in Th2 differentiation and gene expression}

STAT6 and initiation of Th2 differentiation. The signal transducer and activator of transcription (STAT) proteins play an important role in the selective response of cells to particular cytokines (32). After stimulation with cytokines, STAT proteins undergo phosphorylation by the Janus family of kinases (Jak). This causes dimerization and nuclear translocation of the proteins, wherein they induce transcription of target genes. IL-4 engagement with its receptor leads to phosphorylation of STAT 6 by Jak 1 and Jak3, and results in the activation of IL-4-regulated genes such as IL-4R, IgE, FcR, and MHC class II molecules (32). An alternate pathway for STAT6, particularly in humans, is through IL-13 (33). Whereas $I L-4^{-/-}$mice have residual Th2 responses, STAT6 ${ }^{-/-}$mice have almost complete abolition of Th2 responses. In murine models of allergic inflammation, STAT6 ${ }^{-/-}$mice showed marked attenuation of pulmonary eosinophilia, mucus production, AHR and serum IgE levels.

GATA-3: a key regulator of T-cell development, Th2 differen- 


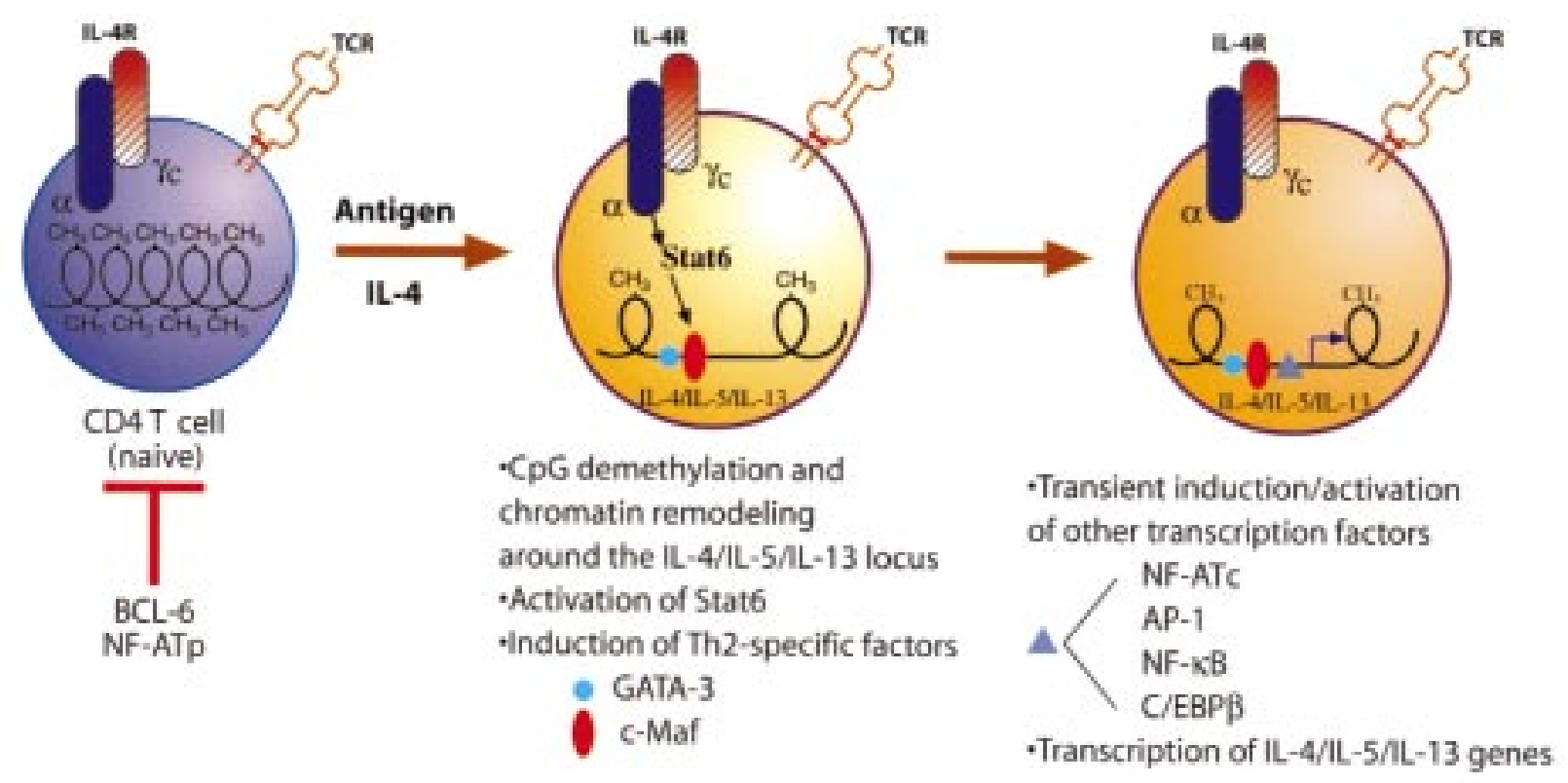

Figure 2

Molecular mechanisms of differentiation of a naive $C D 4^{+} T$ cell into Th2 cells. A naive $C D 4^{+} T$ cell contains a condensed chromatin structure with extensive methylation. Antigen stimulation and engagement of the IL-4R result in STAT6 activation, which, in turn, causes specific demethylation around the IL-4/IL-5/IL-13 locus (similarly, antigen + IL-12 causes demethylation around the IFN- $\gamma$ locus). Chromatin remodeling is accompanied by induction of Th2-specific transcription factors such as GATA-3 and c-Maf, which bind to target sequences in the IL-4/IL-5/IL-13 locus. The chromatin - rendered accessible by demethylation and perhaps by binding of GATA-3, c-Maf, and other, currently undiscovered Th2-specific transcription factors - is next bound by more widely expressed and transiently induced transcription factors such as AP-1, NF- KB, NF-ATc, and C/EBP $\beta$. This may allow synergistic interactions between the tissue-specific and general transcription factors to occur, resulting in the active transcription of the IL-4, IL-5, and IL-13 genes. Effector/memory cells are thought to be in a state of suspended animation, with an open chromatin structure and high levels of GATA-3 and c-Maf expression. Restimulation of these cells by antigen would result in transient induction of the general factors leading to rapid induction of Th2 gene expression.

tiation, and the Th1/Th2 balance. GATA-3 was first described as a transcription factor that interacted with the TCR- $\alpha$ gene enhancer (34). GATA-3 belongs to the GATA family of transcription factors, which bind to the WGATAR (W $=\mathrm{A} / \mathrm{T} ; R=\mathrm{A} / \mathrm{G})$ DNA sequence through a highly conserved $\mathrm{C}_{4}$ zinc-finger domain. Six members (GATA-1 to GATA-6) of this family have been identified in avians, with homologues in mammals and avians. Based on their expression profile and structure, the GATA proteins may be classified as hematopoietic (GATA-1 to GATA-3) (35) or nonhematopoietic (GATA-4 to GATA-6).

Targeted disruption of the GATA-3 gene in mice results in embryonic death on day 12 , with a failure of fetal liver hematopoiesis and defects in the central nervous system (36). The lethal effects of GATA-3 deficiency were bypassed by generating GATA-3-/- chimeras and by using antisense oligonucleotides for GATA-3 in fetal thymus organ cultures $(37,38)$. These elegant studies established an essential role for GATA-3 in the earliest steps of T-cell development. The targets of GATA-3 in the early T-cell progenitors are presently unknown, yet these initial studies clearly demonstrated that GATA-3 is not a functionally redundant GATA family protein.

In addition to its essential role in T-cell development, GATA-3 has also been identified as a Th2 differentiation factor. Naive $\mathrm{CD} 4^{+} \mathrm{T}$ cells express low levels of GATA-3 mRNA $(39,40)$. The expression of GATA-3 is, however, markedly upregulated in cells differentiating along the Th2 lineage, and is downregulated in cells differentiating along the Th1 pathway $(39,40)$. A role for GATA-3 in the expression of a Th2 cytokine gene was first established by Siegel et al., who demonstrated the critical importance of a GATA3 binding site in the IL- 5 promoter (41). Subsequently, by using established clones as well as Th1 or Th2 cells generated from naive $\mathrm{CD}^{+} \mathrm{T}$ cells (39), and by using representational differential analysis (42), Th2-specific expression of the GATA-3 gene was demonstrated. In further studies, GATA-3 was shown to be sufficient for activation of the IL5 , but not IL-4, promoter in a non-Th2 environment (43, 44). Other than the IL-5 gene, a functional GATA-3 binding site has yet to be identified in Th2-expressed cytokine genes. However, several regions around the IL-4/IL-13 locus have been shown to bind GATA-3 (44), the functional implications of which remain to be determined.

To investigate the functional significance of downregulation of GATA-3 gene expression in Th1 cells, GATA3 was forcibly overexpressed in Th1 cells. In developing Th1 cells, overexpression of GATA-3 resulted in abolition of IL-12R $\beta_{2}$ subunit expression with concomitant abrogation of IFN- $\gamma$ production (40). In previously polarized Th1 effector cells, GATA-3 overexpression did not inhibit IFN- $\gamma$ gene expression (40). This suggests that GATA-3 does not directly act on the IFN- $\gamma$ promoter, but has indirect effects on activated $\mathrm{CD}^{+} \mathrm{T}$ cells, shifting their development away from Th1. Thus, GATA-3 controls Th2 activity by inducing Th2 cytokine gene expression and by biasing Th1/Th2 balance in vivo.

GATA-3 in asthma. In keeping with the crucial role of 
Th2 cells in asthma, a significant increase in GATA-3 expression was demonstrated in asthmatic airways compared with that in control subjects (45). The increase in GATA-3 expression in the asthmatic subjects correlated significantly with IL-5 expression and AHR (45).

While the studies in humans clearly showed increased GATA-3 expression in asthma, the initial animal studies of GATA-3 in Th2 gene expression did not conclusively indicate that GATA-3 was sufficient for the expression of all Th2 cytokine genes. Thus, it was unclear whether GATA-3 inhibition would block the production of all the key Th2 cytokines that have been implicated in asthma, i.e., IL-4, IL-5, and IL-13. Because deficiency of the GATA3 gene causes embryonic lethality, transgenic mice expressing a dominant-negative mutant of GATA-3 in an inducible and $\mathrm{T}$ cell-specific fashion were developed to address this question. These studies demonstrated that inhibition of GATA-3 activity causes a severe blunting of Th2 effects, both locally in the lung (eosinophil influx and mucus production) and systemically (IgE production) (46). Although the precise mechanisms by which GATA-3 controls expression of all the Th2 cytokine genes is presently unknown, it is possible that in addition to directly transactivating the IL-5 promoter, it also acts as a chromatin remodeling factor, allowing the transcription of IL-4 and IL-13. Taken together, studies of GATA3 in mice and humans provide a basis for targeting this important regulator of Th2 gene expression in therapies of asthma and allergic diseases.

The role of $c-M a f$ (Th2-specific), NF-AT, AP-1, C/EBP $\beta$, and $N F-\kappa B$ in Th2 gene expression. Because IL-4 is a potent stimulus and a critical factor for Th2 cell generation, IL-4 gene regulation has been intensely studied in many laboratories as a model system for Th2 gene expression. The inability of GATA- 3 to activate the IL-4 promoter and support IL-4 production in a non-Th2 environment suggests that there is a requirement for additional transcription factors that probably act in concert with GATA-3 to activate IL-4 gene expression. In a study by Ho et al., the transcription factor c-Maf was identified as a Th2-specific factor and was shown to transactivate the IL-4 promoter (47). Studies of $c-\mathrm{Maf}^{\prime-}$ mice recently reported by Kim et al. also demonstrate a crucial role for c-Maf in IL-4 gene expression (48). Whereas $c-\mathrm{Maf}^{-1}$ mice displayed impaired IL-4 production by $\mathrm{CD}^{+}{ }^{+} \mathrm{T}$ cells, production of IL- 5 and IL-13 was normal in these animals (48). Also, upon immunization, $c-M a f^{\prime-}$ mice expressed normal IgE levels, which was probably due to unimpaired IL-13 production. Therefore, unlike GATA-3, c-Maf directly activates the IL-4 promoter, but does not appear to regulate the expression of all Th2 cytokine genes (48).

NF-AT has also been shown to directly activate the IL-4 promoter. NF-AT proteins contain a cytoplasmic subunit and an inducible nuclear component. Four related genes encoding the cytoplasmic subunit are currently known: NF-ATp (NF-AT1), NF-ATc (NF-AT2), NF-AT3 and NFAT4. NF-AT proteins are present in both Th1 and Th2 cells, and have been implicated in the expression of both Th1 and Th2 genes in in vitro studies (49). However, studies of mice genetically deficient in specific NF-AT members have revealed a $\mathrm{Th} 2$-selective role of these proteins in $\mathrm{CD}^{+} \mathrm{T}$-cell responses. While NF-ATp-deficient mice are viable (50), NF-ATc deficiency causes embryonic lethality (50). In studies of immune responses in $\mathrm{NF}-A \mathrm{Ac}^{-/-}$ chimeric mice, severe T-cell defects in IL-4 production and Th2 differentiation were observed (50).

Other transcription factors that are more generally expressed, such as NF- $\mathrm{KB}, \mathrm{C} / \mathrm{EBP} \beta$, and $\mathrm{AP}-1$, are also important for Th2 gene expression. The transcription factor NF- $\mathrm{KB}$ belongs to the Rel family of proteins, and the classic NF- $\kappa B$ complex is a heterodimer composed of 2 polypeptide subunits: p50 and RelA (p65). Studies of mice with targeted disruptions of specific Rel proteins show that these proteins are not functionally redundant. For example, while p65 deficiency caused embryonic lethality in mice, $p 50^{-/-}$mice developed normally. However, when $p 50^{-1-}$ mice were tested in a murine model of allergic airway inflammation, no airway inflammation was induced in these mice. Strikingly, the $p 50^{-/-}$mice failed to express IL-5, which is important for the proliferation and differentiation of eosinophils, or eotaxin, which is important for the recruitment of eosinophils into the asthmatic airway (51). The transcription factors $\mathrm{C} / \mathrm{EBP} \beta$ and $\mathrm{AP}-1$, which are widely expressed in many cell types, have been also shown to be important for the expression of Th2 cytokine genes $(41,50)$. Collectively, these studies emphasize that while the Th2-specific factors, such as GATA-3 and c-Maf, are essential and determine the tissue specificity of gene expression, they are not sufficient for optimal expression of the cytokine genes. The concomitant activation of more general factors, such as NF- $\kappa \mathrm{B}, \mathrm{AP}-1, \mathrm{NF}-\mathrm{AT}$ and $\mathrm{C} / \mathrm{EBP} \beta$, is required for highlevel expression of the Th2 cytokine genes.

Negative regulators of Th2 gene expression: $B C L-6$ and NF-

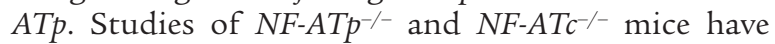
revealed an interesting reciprocal relationship between the 2 proteins in the regulation of Th 2 responses. Whereas NF-ATc ${ }^{-1-}$ chimeric mice displayed impaired IL-4 production, $N F-A T P^{-1-}$ mice showed exaggerated and prolonged IL-4 production and constitutive nuclear localization of NF-ATc (50). The mice displayed increased Th 2 and decreased Th 1 responses both in vivo and in vitro. Thus, unlike NF-ATc, NF-ATp can behave as a negative regulator of Th2 responses in vivo.

Another molecule that appears to be a repressor of Th2 responses in vivo is BCL-6, which was originally identified as a proto-oncogene that is frequently translocated in diffuse large-cell lymphoma. BCL-6 is a potent transcriptional repressor, and has been shown to bind the STAT6 DNA-binding sequence in the CD23b promoter and to repress IL-4-induced activation of CD23 expression (52). Interestingly, targeted deletion of the BCL-6 gene led to a massive inflammatory response in many organs (including the lung), a response characterized by eosinophilic infiltration $(52,53)$. IgE responses were also elevated in immunized BCL-6-deficient mice $(52,53)$. Although the precise mechanism by which BCL-6 represses Th2 responses is unclear, dysregulation of STAT-responsive gene expression most likely underlies the aberrant inflammatory response in BCL-6-deficient mice. Interestingly, mice lacking STAT6 and BCL-6, or mice lacking both BCL- 6 and IL-4, were shown to mount a Th2 response upon antigen provocation in vivo (54). Therefore, Th2 cell generation can be independent of IL- 
4 and STAT6. The factors that control this alternate pathway have not yet been identified.

The hierarchy of transcription factors and differential control in Th2 gene expression. The pattern of expression of the various transcription factors during Th2 gene expression suggests a hierarchy of control that determines coordinate or differential expression of the Th2 cytokine genes. The precise relationship between STAT6, GATA-3, NFAT, and c-Maf is not clear. However, the initiation of Th2 differentiation in antigen- and cytokine-stimulated naive CD $4^{+}$T cells appears to be orchestrated by STAT6, which preexists in the cell cytoplasm. NF-ATp and NF-ATc also exist in the cytoplasm of naive $\mathrm{CD} 4^{+} \mathrm{T}$ cells, and undergo rapid nuclear translocation upon stimulation of the cells. However, their precise positions in the activation cascade are undefined. The expression of both GATA-3 and c-Maf is clearly induced in developing Th2 cells, albeit with different kinetics. While GATA-3 expression peaks at 48 hours after stimulation $(39,40)$, the expression of c-Maf peaks between 5 and 7 days after antigenic stimulation (47). Are both factors required for the expression of all the Th2 cytokines? As discussed above, neither GATA-3 nor c-Maf is sufficient, but both are critical for IL-4 gene expression in vivo. However, GATA-3 plays an essential role in the control of production of all key Th2 cytokines implicated in asthma pathogenesis (46). Analysis of genetically manipulated mice has also revealed how the transcription of individual Th2 cytokine genes is differentially controlled. For example, NF- $\mathrm{KB}$ appears to be more important for antigen-induced IL-5 gene expression than for IL-4 gene expression (51). In contrast, c-Maf is a critical regulator of IL-4, but not IL-5 or IL-13, gene expression (48). GATA-3, on the other hand, regulates the expression of all of these genes (46). These different levels of molecular control might explain differential expression of Th2 cytokines in particular situations. There is increasing interest in the dissociation of IL-4 and IL-5 gene expression in $\mathrm{T}$ cells in various disease situations and in T-cell lineages. In intrinsic asthmatics, increased IL-5, but not IL-4, production has been reported (5). In contrast, in leukemic Sézary cells, IL-4 production is upregulated but IL-5 production is downregulated (55). Although there is no evidence to support different Th cell lineages for IL-4 and IL-5 production, it is reasonable to speculate that once cells have been committed to the Th2 lineage, different microenvironmental factors might activate different sets of transcription factors, such as GATA3 , c-Maf, NF-AT, and NF- $\mathrm{KB}$, to cause differential expression of cytokine genes.

Cell-intrinsic mechanisms and probabilistic gene expression in $\mathrm{CD}^{+} \mathrm{T}$ cells. The studies noted above describe the processes that regulate Th2 gene transcription, which may influence the amount of cytokine produced on a per cell basis. Recent studies have shown that the intensity of a Th2 response can also be regulated by the number of cells making Th2 cytokines (IL-4), without influencing the amount produced by individual $\mathrm{CD}^{+} \mathrm{T}$ cells. This alternate genetically controlled pathway of probabilistic gene activation has been proposed to explain the greater Th2 bias in BALB/c mice compared with C57BL/ 6 mice. As a result, a greater number of $\mathrm{CD} 4^{+} \mathrm{T}$ cells are committed to $\mathrm{IL}-4$ production in the $\mathrm{BALB} / \mathrm{c}$ animals. Interestingly, this regulatory event occurs upstream of cytokine-mediated effects that induce expression of Th2-specific factors such as GATA-3 and c-Maf (56). Another unexpected finding in cytokine gene expression that is consistent with probabilistic gene expression is monoallelic expression of cytokine genes (for IL-2, IL-4, and probably others), which may be due to differential chromatin remodeling at independent alleles. The potential importance of this regulation for human diseases such as asthma and atopy is obvious but remains to be explored in the future.

Thus, the key intracellular events that orchestrate Th2specific gene expression upon antigen and cytokine stimulation of naive $\mathrm{CD}^{+} \mathrm{T}$ cells include chromatin remodeling accompanied by induction of Th2-specific factors such as GATA-3 and c-Maf (Figure 2). These initial events help maintain a stable Th2 phenotype in the committed cells. Th2-specific factors synergize with other, more generally expressed factors (NF-AT, NF- $\mathrm{B}, \mathrm{AP}-1$ ) that are transiently induced during this process, resulting in cytokine (IL-4, IL-5, IL-13) gene transcription. Superimposed on this process is a genetically controlled mechanism that appears to determine the fraction of $\mathrm{CD}^{+} \mathrm{T}$ cells that are committed to the Th2 lineage and the specific allele that is expressed in these cells.

Treatment of asthma. The impressive data implicating Th2 cell activation in the pathogenesis of asthma support a range of new therapeutic strategies that would reduce Th2-induced inflammation and its consequences in the airways. These interventions can be aimed at inhibiting Th 2 cell effects at 3 different points in the inflammatory cascade in asthma (Figure 3): (a) Th2 cell differentiation can be blocked, thus inhibiting the generation of Th2 cells; (b) Th2 activation can be blocked and cytokine secretion inhibited; and (c) Th2 cytokine effects can be blocked at the target tissue.

Blockade of Th2 cell differentiation. Altering the cytokine environment that is present during $\mathrm{CD} 4^{+} \mathrm{T}$-cell differentiation can inhibit the development of new Th2 cells. Blocking IL-4, IL-13, and the molecular signaling mechanisms activated by engagement of IL-4R $\alpha$ (STAT6) has been shown to inhibit the generation of Th2 cells in animals (10). As discussed in this review, GATA-3 blockade by overexpression of a dominant-negative mutant inhibited Th2 cell induction (46). In many cases, blockade of Th2 cell differentiation results in the generation of a Th1 cell population. An alternative method to reduce Th2 cell generation in the respiratory tract is to increase levels of IL-12 and IFN- $\gamma$ in the environment, thus shifting the Th1/Th2 balance toward Th1. Recent epidemiologic evidence suggests that prior infection with M. tuberculosis or measles is associated with a reduction in atopy and asthma (57). In mice infected with Bacille CalmetteGuérin (BCG) prior to antigen sensitization, there was a reduction in eosinophilic airway inflammation and AHR (58). These studies imply that altering the cytokine milieu in the respiratory tract by stimulating a Th1 response to certain infectious agents can reduce symptoms in asthma. The administration of CPG oligonucleotides to mice at the time of antigen sensitization also resulted in skewing of the inflammatory response toward Th1, possibly by stimulating sustained IL-12 production (59). This led to a reduction in eosinophilia and 
AHR. All of these interventions may have an effect in asthma by reducing the number of Th 2 cells in the airways. Even when Th 2 cells are already established in the airway, altering the cytokine environment by Th2 cytokine blockade or by stimulating Th1 cell induction may inhibit the generation of new Th2 cells.

Blockade of Th2 cell activation. Glucocorticoids are currently the mainstay of therapy in asthma, and lead to effective control of symptoms by reducing airway inflammation. Steroid treatment leads to a reduction in cytokines, chemokines, cell adhesion molecules, and DCs in the respiratory tract. All of these effects are believed to contribute to the suppression of the inflammatory response in asthma. Therapy with glucocorticoids results in a reduction in total $\mathrm{CD} 4^{+} \mathrm{T}$ cells in the airways and a decrease in mRNA for IL-4, IL-5, and IL13 , yet also results in increased IFN- $\gamma$ expression (60). These effects likely indicate an increased sensitivity to steroid treatment of IL-4, IL-5, and IL-13 genes compared with IFN- $\gamma$. It is unclear what role this shift from Th2 to Th1 plays in the therapeutic effects of steroids, as this is only one of many possible mechanisms by which glucocorticoids can influence inflammation in asthma.

What is clear is that systemic glucocorticoids cause gen- eral immunosuppression and many well-described side effects. These effects can be reduced by local steroid treatment, but the suppression of airway inflammation is only temporary. Thus, selective approaches that block Th2 cell activation are needed. If selective inhibition of Th2 cell activation can be achieved, it will reduce Th2 cytokine secretion, without compromising Th1 cell function. By blocking cytokine secretion, not only will the effects on target tissues be reduced, but the cytokine environment may be altered, thus reducing the generation of new Th2 cells. GATA-3 is T cell-specific and, at present, the only identified Th2-specific factor that has the potential to inhibit the production of all the key Th2 cytokines (46). Blockade of GATA-3 may therefore inhibit Th2 cytokine secretion and also block the generation of new Th2 cells, as described above. Although some studies have shown that Th1 cells, through the production of IFN- $\gamma$, can inhibit Th2 cell function in vitro, these specific effects have not been demonstrated in vivo in animals.

Blockade of Th2 cytokine effects. Blocking the effects of Th2 cytokines at the target tissue is another potential option for reducing the inflammatory effects of Th 2 cells. The effects of cytokine blockade may not only have local influence on airway epithelial cells and on the vascular

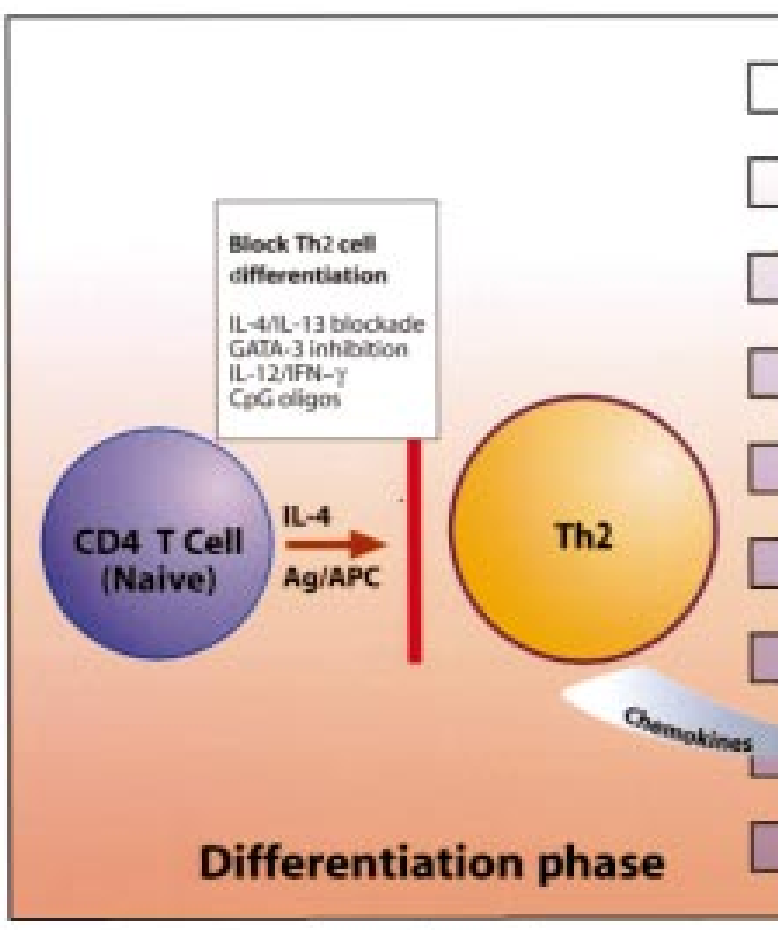

Lymph node

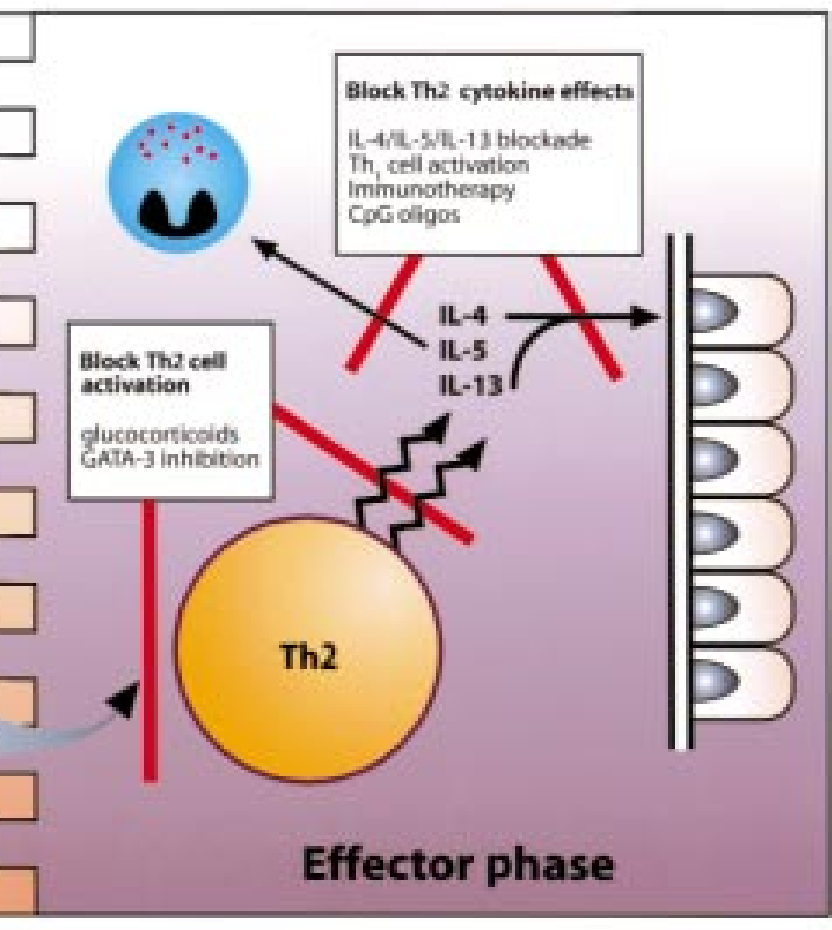

Lung

\footnotetext{
Figure 3

Potential strategies for inhibiting Th2 function in asthma. (a) Block Th2 cell differentiation. The cytokine environment that is present during CD4 T-cell differentiation affects the development of new Th2 cells. Blocking IL-4 and/or IL-13 or the Th2-specific transcription factor GATA-3 leads to inhibition of Th 2 cell induction. Alternatively, increasing levels of IL-12 and IFN- $\gamma$ in the environment may shift the Th1/Th2 balance toward Th1 and reduce the number of Th 2 cells in the respiratory tract. This can be accomplished by stimulating a Th1 response to certain infectious agents, such as Mycobacterium, or by the administration of $\mathrm{CpG}$ oligodeoxynucleotides. All of these interventions may have an effect in asthma by reducing the number of Th 2 cells in the airways and by inhibiting the generation of new Th2 cells. (b) Block Th2 cell activation. Glucocorticoids are standard therapy in asthma and cause nonselective immunosuppression. Th2 cell activation may be selectively blocked using inhibitors of GATA-3, a T cell-specific factor that controls the production of key Th2 cytokines. (c) Block Th2 cytokine effects. Blocking IL-4, IL-5, or IL-13 may inhibit the effects of these cytokines on target tissues. In addition, Th1 cells have been shown to inhibit Th2 cytokine effects on eosinophils and airway epithelial mucus production. Allergy immunotherapy and CpG oligodeoxynucleotides both induce Th1 cells and lead to a reduction in allergic airway pathology.
} 
endothelium, but also may reduce systemic effects on the bone marrow eosinophilopoiesis and on B-cell immunoglobin production. We have recently shown that when both Th1 and Th 2 cells are transferred into recipient mice and activated in the respiratory tract with inhaled antigen, Th1 effects predominate, resulting in decreased airway eosinophilia and mucus production (61). These effects are mediated by IFN- $\gamma$ acting on the target tissue in recipient mice, and occur while Th 2 cells are actively secreting cytokines. In ragweed-sensitized mice, administration of CpG oligodeoxynucleotides, potent inducers of Th1 cells, led to a reduction in ragweed-induced airway eosinophilia, IgE, and AHR (62). This may also be a mechanism by which immunotherapy results in improvement of symptoms, as a reduction in eosinophilia in some allergic patients is associated with an increase in IFN- $\gamma$ levels (63). Thus, Th1 cells, through secretion of IFN- $\gamma$, may block the effects of Th2 cells on target tissues, leading to a reduction in airway pathology.

If our ultimate goal is the long-term reduction in the number and activity of Th2 cells in the respiratory tract, then we must focus on new modes of therapy in asthma. One approach could be to increase the population of activated Th1 cells at sites of allergic inflammation, which may suppress ongoing Th2 cytokine effects. An alternate approach is to selectively target Th2 cells. Targeting Th2-specific factors such as GATA-3 by using pharmacologic or antisense approaches will inhibit Th2 responses while sparing Th1 cells and avoiding general immunosuppression. Over time, it is possible that these effects will be sustained, as there is evidence that potent, long-term stimulation with Th1 cytokines may shift a Th2-predominant population toward Th1 (24). These immunomodulatory approaches have the potential of disturbing the highly regulated immune defense system in the respiratory tract, including increasing unwanted Th1 effects such as autoimmune diseases, delayed-type hypersensitivity reactions, and excessive inflammation (64). The potential value of immunomodulation in controlling asthma, however, demands that these therapies be further developed and tested.

\section{Acknowledgments}

The authors wish to thank David Field for the expert artwork, and Jack Elias, Prabir Ray, and Kim Bottomly for suggestions and critical review of the manuscript. This work was supported by grants HL-56843 and HL-60995 (to A. Ray), P50-HL56389 (to A. Ray and L.Cohn), and HL-03308 (to L. Cohn) from the National Heart, Lung, and Blood Institute of the National Institutes of Health.

1. Wills-Karp, M. 1999. Immunologic basis of antigen-induced airway induced hyperresponsiveness. Annu. Rev. Immunol. 17:255-281.

2. Cohn, L., Homer, R.J., Marinov, A., Rankin, J., and Bottomly, K. 1997. Induction of airway mucus production by T helper 2 (Th2) cells: a critical role for interleukin 4 in cell recruitment but not mucus production. J. Exp. Med. 186:1737-1747.

3. Cohn, L., et al. 1999. Th2-induced airway mucus production is dependent on IL-4Ralpha, but not on eosinophils. J. Immunol. 162:6178-6183.

4. Corrigan, C.J., Hartnell, A., and Kay, A.B. 1988. T lymphocyte activation in acute severe asthma. Lancet. 1:1129-1132.

5. Walker, C., et al. 1992. Allergic and nonallergic asthmatics have distinct patterns of T-cell activation and cytokine production in peripheral blood and bronchoalveolar lavage. Am. Rev. Respir. Dis. 146:109-115.

6. Cher, D., and Mosmann, T. 1987. Two types of murine helper T cell clone. II. Delayed type hypersensitivity is mediated by Th1 clones. $J$. Immunol. 138:3688-3694.

7. Kim, J., Woods, A., Becker-Dunn, E., and Bottomly, K. 1985. Distinct functional phenotypes of cloned Ia-restricted helper T cells. J. Exp. Med. 162:188-201.

8. Mosmann, T.R., Cherwinski, H., Bond, M.W., Giedlin, M.A., and Coffman, R.L. 1986. Two types of murine helper T cell clone. I. Definition according to profiles of lymphokine activities and secreted proteins. J. Immunol. 136:2348-2357.

9. Killar, L., MacDonald, G., West, J., Woods, A., and Bottomly, K. 1987. Cloned Ia restricted T cells that do not produce IL4/BSF-1 fail to help antigen specific B cells. J. Immunol. 138:1674-1679.

10. Barner, M., Mohrs, M., Brombacher, F., and Kopf, M. 1998. Differences between IL-4R alpha-deficient and IL-4-deficient mice reveal a role for IL-13 in the regulation of Th2 responses. Curr. Biol. 8:669-672.

11. Coffman, R.L., Seymour, B.W.P., Hudak, S., Jackson, J., and Rennick, D. 1989. Antibody to interleukin-5 inhibits helminth-induced eosinophilia in mice. Science. 245:308-310.

12. Nakajima, H., et al. 1992. CD4+ T-lymphocytes and interleukin-5 mediate antigen-induced eosinophil infiltration into mouse trachea. Am. Rev. Respir. Dis. 146:374-377.

13. Robinson, D.S., et al. 1992. Predominant TH2-like bronchoalveolar Tlymphocyte population in atopic asthma. N. Engl. J. Med. 326:298-304.

14. Humbert, M., et al. 1997. Elevated expression of messenger ribonucleic acid encoding IL-13 in the bronchial mucosa of atopic and nonatopic subjects with asthma. J. Allergy Clin. Immunol. 99:657-665.

15. Kotsimbos, T.C., Ernst, P., and Hamid, Q.A. 1996. Interleukin-13 and interleukin-4 are coexpressed in atopic asthma. Proc. Assoc. Am. Physicians. 108:368-373.

16. Holtzman, M.J., Sampath, D., Castro, M., Look, D.C., and Jayaraman, S. 1996. The one-two of T helper cells: does interferon-gamma knock out the Th2 hypothesis for asthma? Am. J. Respir. Cell. Mol. Biol. 14:316-318.

17. Cembrzynska-Nowak, M., Szklarz, E., Inglot, A.D., and TeodorczykInjeyan, J.A. 1993. Elevated release of tumor necrosis factor-alpha and interferon-gamma by bronchoalveolar leukocytes from patients with bronchial asthma. Am. Rev. Respir. Dis. 147:291-295.

18. Krug, N., et al. 1996. T cell cytokine profile evaluated at the single cells level in BAL and blood in allergic asthma. Am. J. Respir. Cell. Mol. Biol. 14:319-326.

19. Kelly, E.A., Rodriguez, R.R., Busse, W.W., and Jarjour, N.N. 1997. The effect of segmental bronchoprovocation with allergen on airway lymphocyte function. Am. J. Respir. Crit. Care Med. 156:1421-1428.

20. Renz, H., et al. 1993. Specific V beta $T$ cell subsets mediate the immediate hypersensitivity response to ragweed allergen. J. Immunol. 151:1907-1917.

21. Cohn, L., Tepper, J.S., and Bottomly, K. 1998. IL-4-independent induction of airway hyperresponsiveness by Th2, but not Th1, cells. J. Immunol. 161:3813-3816.

22. Grunig, G., et al. 1998. Requirement for IL-13 independently of IL-4 in experimental asthma. Science. 282:2261-2263.

23. Wills-Karp, M., et al. 1998. Interleukin-13: central mediator of allergic asthma [see comments]. Science. 282:2258-2261.

24. Coffman, R.L., Mocci, S., and O'Garra, A. 1999. The stability and reversibility of Th1 and Th2 populations. Curr. Top. Microbiol. Immunol. 238:1-12.

25. Zuany-Amorim, C., et al. 1998. Requirement for gammadelta T cells in allergic airway inflammation. Science. 280:1265-1267.

26. Kung, T.T., et al. 1995. Mast cells modulate allergic pulmonary eosinophilia in mice. Am. J. Respir. Cell. Mol. Biol. 12:404-409.

27. Stumbles, P.A., et al. 1998. Resting respiratory tract dendritic cells preferentially stimulate $\mathrm{T}$ helper cell type 2 (Th2) responses and require obligatory cytokine signals for induction of Th1 immunity. J. Exp. Med. 188:2019-2031.

28. Rissoan, M.C., et al. 1999. Reciprocal control of T helper cell and dendritic cell differentiation [see comments]. Science. 283:1183-1186.

29. Constant, S.L., and Bottomly, K. 1997. Induction of Th1 and Th2 CD4+ $\mathrm{T}$ cell responses: the alternative approaches. Annu. Rev. Immunol. 15:297-322.

30. Comoy, E., et al. 1999. Do allergens induce type- 2 immune responses? Int. Arch. Allergy. Immunol. 118:399-402.

31. Agarwal, S., and Rao, A. 1998. Modulation of chromatin structure regulates cytokine gene expression during T cell differentiation. Immunity. 9:765-775.

32. Kaplan, M.H., and Grusby, M.J. 1998. Regulation of T helper cell differentiation by STAT molecules. J. Leukoc. Biol. 64:2-5.

33. Palmer-Crocker, R.L., Hughes, C.C., and Pober, J.S. 1996. IL-4 and IL-13 activate the JAK2 tyrosine kinase and Stat 6 in cultured human vascular endothelial cells through a common pathway that does not involve the gamma c chain. J. Clin. Invest. 98:604-609.

34. Ho, I.C., et al. 1991. Human GATA-3: a lineage-restricted transcription factor that regulates the expression of the $\mathrm{T}$ cell receptor alpha gene. EMBO J. 10:1187-1192. 
35. Orkin, S.H. 1995. Hematopoiesis: how does it happen? Curr. Opin. Cell Biol. 7:870-877.

36. Pandolfi, P., et al. 1995. Target disruption of the GATA-3 gene causes severe abnormalities in the nervous system and in fetal liver hematopoiesis. Nat. Genet. 11:40-44.

37. Ting, C.-N., Olson, M.C., Barton, K.P., and Leiden, J.M. 1996. Transcription factor GATA-3 is required for development of the T-cell lineage. Nature. 384:474-478.

38. Hattori, N., Kawamoto, H., Fujimoto, S., Kuno, K., and Katsura, Y. 1996. Involvement of transcription factors TCF-1 and GATA-3 in the initiation of the earliest step of T cell development in the thymus. J. Exp. Med. 184:1137-1147.

39. Zhang, D.-H., Cohn, L., Ray, P., Bottomly, K., and Ray, A. 1997. Transcription factor GATA- 3 is differentially expressed in Th 1 and Th 2 cells and controls Th2-specific expression of the interleukin-5 gene. J. Biol. Chem. 272:21597-21603.

40. Ouyang, W., et al. 1998. Inhibition of Th1 development mediated by GATA-3 through an IL-4-independent mechanism. Immunity. 9:745-755.

41. Siegel, M.D., Zhang, D.-H., Ray, P., and Ray, A. 1995. Activation of the interleukin-5 promoter by cAMP in murine EL-4 cells requires the GATA-3 and CLE0 elements. J. Biol. Chem. 270:24548-24555.

42. Zheng, W., and Flavell, R.A. 1997. The transcription factor GATA-3 is necessary and sufficient for Th2 cytokine gene expression in CD4 T cells. Cell. 89:587-596.

43. Zhang, D.-H., Yang, L., and Ray, A. 1998. Differential responsiveness of the interleukin-5 (IL-5) and IL-4 genes to transcription factor GATA-3. J. Immunol. 161:3817-3821.

44. Ranganath, S., et al. 1998. GATA-3-dependent enhancer activity in IL-4 gene regulation. J. Immunol. 161:3822-3826.

45. Nakamura, Y., et al. 1999. Gene expression of the GATA-3 transcription factor is increased in atopic asthma. J. Allergy Clin. Immunol. 103:215-222.

46. Zhang, D.H., et al. 1999. Inhibition of allergic inflammation in a murine model of asthma by expression of a dominant-negative mutant of GATA-3. Immunity. In press.

47. Ho, I.-C., Hodge, M.R., Rooney, J.W., and Glimcher, L.H. 1996. The proto-oncogene c-maf is responsible for tissue-specific expression of interleukin-4. Cell. 85:973-983.

48. Kim, J.I., Ho, I.-C., Grusby, M.J., and Glimcher, L.H. 1999. The transcription factor c-Maf controls the production of IL-4 but not other Th2 cytokines. Immunity. 10:745-751.

49. Rao, A., Luo, C., and Hogan, P.G. 1997. Transcription factors of the NF-
AT family: regulation and function. Annu. Rev. Immunol. 15:707-747. 50. Glimcher, L.H., and Singh, H. 1999. Transcription factors in lymphocyte development $-\mathrm{T}$ and $\mathrm{B}$ cells get together. Cell. 96:13-23.

51. Yang, L., et al. 1998. Essential role of nuclear factor $\mathrm{kB}$ in the induction of eosinophilia in allergic airway inflammation. J. Exp. Med. 188:1739-1750.

52. Dent, A.L., Shaffer, A.L., Yu, X., Allman, D., and Staudt, L.M. 1997. Control of inflammation, cytokine expression, and germinal center formation by BCL-6. Science. 276:589-592.

53. Ye, B.H., et al. 1997. The BCL-6 proto-oncogene controls germinal-centre formation and Th2-type inflammation. Nat. Genet. 16:161-170.

54. Dent, A.L., Hu-Li, J., Paul, W.E., and Staudt, L.M. 1998. T helper type 2 inflammatory disease in the absence of interleukin 4 and transcription factor STAT6. Proc. Natl. Acad. Sci. USA. 95:13823-13828.

55. Tendler, C.L., et al. 1994. Abnormal cytokine expression in Sezary and adult T-cell leukemia cells correlates with the functional diversity between these T-cell malignancies. Cancer Res. 54:4430-4435.

56. Bix, M., Wang, Z.E., Thiel, B., Schork, N.J., and Locksley, R.M. 1998. Genetic regulation of commitment to interleukin 4 production by a CD4(+) T cell-intrinsic mechanism. J. Exp. Med. 188:2289-2299.

57. Cookson, W.O.C.M., and Moffatt, M.F. 1997. Asthma: an epidemic in the absence of infection. Science. 275:41-42.

58. Erb, K.J., Holloway, J.W., Sobeck, A., Moll, H., and Le Gros, G. 1998. Infection of mice with Mycobacterium bovis-Bacillus Calmette-Guerin (BCG) suppresses allergen-induced airway eosinophilia. J. Exp. Med. 187:561-569.

59. Kline, J.N., et al. 1998. Cutting edge: modulation of airway inflammation by $\mathrm{CPG}$ oligodeoxynucleotides in a murine model of asthma. $J$. Immunol. 160:2555-2559.

60. Robinson, D., et al. 1993. Prednisolone treatment in asthma is associated with modulation of bronchoalveolar lavage cell interleukin-4, interleukin-5, and interferon-gamma cytokine gene expression. Am. Rev. Respir. Dis. 148:401-406.

61. Cohn, L., et al. 1999. Th 1 cells and IFN $\gamma$ regulate allergic airway inflammaion and mucus production. In press.

62. Sur, S., et al. 1999. Long term prevention of allergic lung inflammation in a mouse model of asthma by CPG oligodeoxynucleotides. J. Immunol. 162:6284-6293.

63. Varney, V.A., et al. 1993. Influence of grass pollen immunotherapy on cellular infiltration and cytokine mRNA expression during allergeninduced late-phase cutaneous responses. J. Clin. Invest. 92:644-651.

64. Holt, P.G. 1994. A potential vaccine strategy for asthma and allied atopic diseases during early childhood. Lancet. 344:456-458. 